\title{
The International Code of Virus Classification and Nomenclature (ICVCN): proposal to delete Rule 3.41
}

\author{
Jens H. Kuhn • Sheli R. Radoshitzky • \\ Sina Bavari $\cdot$ Peter B. Jahrling
}

Received: 13 July 2012/ Accepted: 19 July 2012/Published online: 31 August 2012

(C) Springer-Verlag (outside the USA) 2012

\begin{abstract}
It is proposed to delete Rule 3.41 of the International Code of Virus Classification and Nomenclature, which requires the name of a virus taxon to precede the term for the taxonomic unit.
\end{abstract}

\section{Introduction}

The International Committee on Taxonomy of Viruses (ICTV) is the body tasked by the International Union of Microbiological Societies (IUMS) to make decisions on matters of virus classification and nomenclature [1]. ICTV activities are governed by statutes (the Statutes) [2]. One of these Statutes states that classification and nomenclature will be subject to rules (the Rules) set out in an interna-

\footnotetext{
The content of this publication does not necessarily reflect the views or policies of the US Department of the Army, the US Department of Defense or the US Department of Health and Human Services or of the institutions and companies affiliated with the authors. This work was funded by the Joint Science and Technology Office for Chem Bio Defense (proposal \#TMTI0048_09_RD_T) to SB and SRR. JHK performed this work as an employee of Tunnell Consulting, Inc., a subcontractor to Battelle Memorial Institute, and FKM as an employee of Battelle Memorial Institute, under its prime contract with NIAID, under Contract No. HHSN272200200016I.
}

\section{J. H. Kuhn $(\bowtie) \cdot$ P. B. Jahrling}

Integrated Research Facility at Fort Detrick (IRF-Frederick),

Division of Clinical Research (DCR), National Institute of

Allergy and Infectious Diseases (NIAID), National Institutes of

Health (NIH), Fort Detrick, Frederick, MD, USA

e-mail: kuhnjens@mail.nih.gov

S. R. Radoshitzky $\cdot$ S. Bavari

United States Army Medical Research Institute of Infectious

Diseases, Fort Detrick, Frederick, MD, USA tional code (the Code). The most recent version of this International Code of Virus Classification and Nomenclature (ICVCN) is available in the latest, 9th, ICTV Report, which was published in 2011 [3].

The ICVCN is organized in three major parts: Section 1 outlines the "Statutory basis for the International Committee on Taxonomy of Viruses (ICTV)". Section 2 describes the "Principles of nomenclature". Finally, section 3 outlines the "Rules of Classification and Nomenclature" [capitalization or lack thereof is identical to the headlines in the code] [3].

In contrast to the International Code of Zoological Nomenclature (ICZN Code; http://iczn.org), the International Code of Nomenclature of Bacteria (ICNB; http://www. ncbi.nlm.nih.gov/books/NBK8817/), and the International Code of Botanical Nomenclature (ICBN; http://ibot.sav.sk/ $\mathrm{icbn} / \mathrm{main} . \mathrm{htm}$ ), the ICVCN is rather short and still relatively imprecise. This article is the first of a series of manuscripts that outlines suggestions and proposals that would make the ICVCN a more precise instrument for virus taxonomy, while at the same time decreasing currently existing confusion among laboratory virologists regarding the use of the Rules put forth in the Code.

\section{Proposal to delete Rule 3.41}

ICVCN Rule 3.41 is the last Rule of the Code, located in Subsection IX ("Rules for Orthography") of Section 3 ("Rules of Classification and Nomenclature"). It states

"In formal usage, the name of the taxon shall precede the term for the taxonomic unit"

and is followed by a comment that is not part of the official rule: 
Table 1 Usage of taxon names and taxonomic units

\begin{tabular}{|c|c|c|c|}
\hline & $\begin{array}{l}\text { Correct usage of taxon names and } \\
\text { taxonomic units according to Rule } 3.41 \\
\text { (ignoring the Comment that explains it) }\end{array}$ & $\begin{array}{l}\text { Correct usage of taxon names and taxonomic } \\
\text { units according to the Comment that } \\
\text { explains Rule } 3.41 \text { (ignoring the actual Rule) }\end{array}$ & $\begin{array}{l}\text { Incorrect usage of taxon names and } \\
\text { taxonomic units according to both Rule } \\
3.41 \text { and the Comment that explains it }\end{array}$ \\
\hline \multirow[t]{4}{*}{ Orders } & Xyzvirales order & order Xyzvirales & (the) Xyzvirales \\
\hline & - e.g. Caudovirales order & - e.g. order Caudovirales & - e.g. (the) Caudovirales \\
\hline & - e.g. Mononegavirales order & - e.g. order Mononegavirales & - e.g. (the) Mononegavirales \\
\hline & - e.g. Nidovirales order & - e.g. order Nidovirales & - e.g. (the) Nidovirales \\
\hline \multirow[t]{4}{*}{ Families } & Xyzviridae family & family Xyzviridae & (the) Xyzviridae \\
\hline & - e.g. Filoviridae family & - e.g. family Filoviridae & - e.g. (the) Filoviridae \\
\hline & - e.g. Picornaviridae family & - e.g. family Picornaviridae & - e.g. (the) Picornaviridae \\
\hline & - e.g. Tymoviridae family & - e.g. family Tymoviridae & - e.g. (the) Tymoviridae \\
\hline \multirow[t]{4}{*}{ Subfamilies } & Xyzvirinae subfamily & subfamily Xyzvirinae & (the) Xyzvirinae \\
\hline & - e.g. Densovirinae subfamily & - e.g. subfamily Densovirinae & - e.g. (the) Densovirinae \\
\hline & - e.g. Picovirinae subfamily & - e.g. subfamily Picovirinae & - e.g. (the) Picovirinae \\
\hline & - e.g. Pneumovirinae subfamily & - e.g. subfamily Pneumovirinae & - e.g. (the) Pneumovirinae \\
\hline \multirow[t]{4}{*}{ Genera } & Xyzvirus genus & genus Xyzvirus & Xyzvirus \\
\hline & - e.g. Arenavirus genus & - e.g. genus Arenavirus & - e.g. Arenavirus \\
\hline & - e.g. Begomovirus genus & - e.g. genus Begomovirus & - e.g. Begomovirus \\
\hline & - e.g. Ictalurivirus genus & - e.g. genus Ictalurivirus & - e.g. Ictalurivirus \\
\hline \multirow[t]{4}{*}{ Species } & $\begin{array}{l}\text { Xyz, virus/Xyz abcvirus/Xyzvirus } \\
X \text { species }\end{array}$ & $\begin{array}{l}\text { species } X y z \text { virus/Xyz abcvirus/ } \\
\text { Xyzvirus } X\end{array}$ & $\begin{array}{l}\text { Xyz virus/Xyz abcvirus/ } \\
\text { Xyzvirus } X\end{array}$ \\
\hline & - e.g. Aichi virus species & - e.g. species Aichi virus & - e.g. Aichi virus \\
\hline & - e.g. Marburg marburgvirus species & - e.g. species Marburg marburgvirus & - e.g. Marburg marburgvirus \\
\hline & - e.g. Rotavirus A species & - e.g. species Rotavirus $A$ & - e.g. Rotavirus A \\
\hline
\end{tabular}

"Comments: For example, the correct formal descriptions of various taxa are ... the family Herpesviridae, ...the genus Morbillivirus, .... the genus Rhinovirus, .... the species Tobacco necrosis virus D, and so on".

An official proposal ("TaxoProp") to delete this Rule (TaxoProp No. 2012.001aG.U.v1.delete_Rule_3.41.pdf) was submitted on July 5, 2012, to the ICTV and provisionally approved (http://talk.ictvonline.org/files/proposals/ taxonomy_proposals_general1/m/gen02/4333.aspx) for three reasons.

First, the Comment contradicts the Rule it is supposed to explain. The Rule states that the name of the taxon should precede the term for the taxonomic unit, but the comment suggests the opposite (Table 1).

Second, it is unnecessary in a set of taxonomic rules since it is purely stylistic. To the best of our knowledge, there is no difference in meaning between, for instance, "the Xyzviridae family", "the family Xyzviridae" and "(the) Xyzviridae" (with Xyz being a placeholder for any ICTV-accepted family name). In fact, the term "family" in this context is redundant and therefore stylistically unpleasant because the formal suffix, -viridae, denotes family. The same is true at all taxonomic levels (Table 2). Formal taxon suffixes are widely accepted and well known
Table 2 Taxon-specific suffixes accepted by the ICTV

\begin{tabular}{llll}
\hline Taxon & $\begin{array}{l}\text { Taxon- } \\
\text { specific } \\
\text { suffix }\end{array}$ & $\begin{array}{l}\text { General } \\
\text { usage }\end{array}$ & Examples \\
\hline Order & -virales & Xyzvirales & Caudovirales \\
Family & -viridae & Xyzviridae & Filoviridae \\
Subfamily & -virinae & Xyzvirinae & Densovirinae \\
Genus & -virus & Xyzvirus & Arenavirus \\
Species & virus & Xyz virus & Aichi virus \\
& -virus & Xyzabcvirus* & Marburg marburgvirus \\
& -virus & Xyzvirus $X$ & Rotavirus A \\
\hline
\end{tabular}

* with $a b c$ being a placeholder for the word stem of the name of the genus that includes the species

among virologists. It is therefore self-evident that Xyzvirales refers to an order, Xyzviridae refers to a family, Xyzvirinae refers to a subfamily, Xyzvirinae refers to a genus and $X y z$ virus refers to a species.

Third, the Rule is not observed in any consistent manner by the general virology community, including members of ICTV Study Groups and possibly even ICTV members. Sentence fragments such as "the Xyzvirales/Xyzviridael Xyzvirinae/Xyzvirus/Xyz virus" (name of the taxonomic unit, "order/family/subfamily/genus/species," is missing) 
and "the Xyzvirales/Xyzviridae/Xyzvirinae/Xyzvirus/Xyz virus order/family/subfamily/genus/species" and "the order/family/subfamily/genus/species Xyzvirales/Xyzviridae/ Xyzvirinae/Xyzvirus/Xyz virus" are found in the published literature equally often. None of these three usages differ in information content from the others.

In conclusion, the deletion of Rule 3.41 will bring the Code into line with current practice without adversely affecting taxonomic precision. Deletion of the Rule is also straightforward, as other parts of the Code are not adversely affected by doing so. Since Rule 3.41 is the last Rule of the Code, its deletion would not require renumbering of other Rules and would only require deletion of a reference to it in the Comment to Rule 3.2 ("The universal virus classification system shall employ the hierarchical levels of Order, Family, Subfamily, Genus, and Species"). Once the Rule is deleted, numerous manuscripts will be retrospectively in compliance with the Code, and errors will be avoided prospectively.

\section{References}

1. King AMQ, Adams MJ, Carstens EB, Lefkowitz EJ (eds) (2011) Virus Taxonomy - Ninth Report of the International Committee on Taxonomy of Viruses. Elsevier/Academic Press, London

2. The International Committee on Taxonomy of Viruses (ICTV) (2011) The Statutes of the ICTV. In: King AMQ, Adams MJ, Carstens EB, Lefkowitz EJ (eds) Virus Taxonomy - Ninth Report of the International Committee on Taxonomy of Viruses. Elsevier/ Academic Press, London, pp 1269-1272

3. The International Committee on Taxonomy of Viruses (ICTV) (2011) The International Code of Virus Classification and Nomenclature. In: King AMQ, Adams MJ, Carstens EB, Lefkowitz EJ (eds) Virus Taxonomy-Ninth Report of the International Committee on Taxonomy of Viruses. Elsevier/Academic Press, London, pp 1273-1277 